\title{
Coordination of Charging Plug-in Electric Vehicles (PEV) in Electric Distribution Networks to Minimize Power Losses and Voltage Deviations
}

\author{
Lutfir Rahman Aliffianto \\ Electrical Engineering \\ Department \\ Brawijaya University \\ Malang, Indonesia
}

\author{
Hadi Suyono \\ Electrical Engineering \\ Department \\ Brawijaya University \\ Malang, Indonesia
}

\author{
Harry Soekotjo Dachlan \\ Electrical Engineering \\ Department \\ Brawijaya University \\ Malang, Indonesia
}

\begin{abstract}
Plug-in Electric Vehicle (PEV) is a vehicle with a drive in the form of an electric motor and gets its energy source from a rechargeable battery. PEV (grid-to-vehicle, or G2V) charging activity can pose a risk to the distribution system when PEV is injected into the system. Charging PEV in the distribution system will cause the demand for electrical power to increase. Without a proper and optimal charging coordination system for the many PEVs that exist in the distribution network system, the following technical problems will arise in the network such as: occurrence of unusual and unwanted peak loads in power consumption, voltage sag, transformers or conductors overload, as well as the occurrence of high power system losses in the distribution system. This study discusses the optimization of PEV charging coordination scheduling in a distribution system by considering power losses and voltage deviations. To optimize the coordination of PEV charging, this study used a metaheuristic method called the Binary Particle Swarm Optimization (BPSO) which was then compared with the Binary Gray Wolves Optimization (BGWO) method. This scheme is simulated on the $20 \mathrm{kV}$ Pujon Feeder distribution system in Malang, Indonesia. The initial simulation results, at the time when the most loads were presented, showed 80 percent of PEV penetration, increase in power losses of 54.51 percent and voltage deviation of 9.82 percent. By using the BPSO method, the results showed power loss increase of 27.2 percent and voltage deviation of 7.75 percent. Meanwhile, by using the BGWO method the increase in power losses was 31.09 percent and the voltage deviation was 7.98 percent.
\end{abstract}

Keywords: plug-in electric vehicle; optimization; charging coordination; distribution network; power loss

\section{INTRODUCTION}

Plug-in Electric Vehicle (PEV) is a vehicle with an electric motor which gets energy from a rechargeable battery. Therefore, this vehicle produces low emissions which reduce pollution. PEV can be a more promising option as a way to reduce emissions of greenhouse gases (GHGs). In addition, PEV's lower daily operating costs compared to conventional vehicles with internal combustion engines have attracted the attention of vehicle users [2]. In the last decade, PEV vehicles have developed a very substantial industry in developed countries such as the United States, Japan, and Europe. Several well-known brands of vehicle manufacturers have also started to market several types of plug-in electric vehicles (PEV) globally, including to the Indonesian people.

Basically PEV utilizes a large battery capacity and requires charging to provide sufficient energy as a source of energy for high-power electric motors. PEV batteries can be charged at home or other commercial locations through charging stations in public facilities [3]. PEV is considered as an additional electrical load on the distribution system. PEV (grid-to-vehicle, or G2V) charging activity can pose a risk to the distribution system when injected into the system. Charging PEV in the distribution system will cause the demand for electrical power to increase. Without a proper and optimal charging coordination system for the many PEVs that exist in the distribution network system, technical problems will arise in the network such as: occurrence of unusual and unwanted peak loads in power consumption, occurrence of voltage sag, overloading of transformers or conductors, as well as the occurrence of high power system losses in the distribution system [4]. Therefore, an optimal PEV charging coordination system is needed to avoid these problems.

To overcome the problems when PEV is injected into the distribution system, increasing the power generation capacity is a feasible solution but it is not economical because the required infrastructure is too large. Another available solution is smart grid deployment which can increase system reliability by coordinating PEV charging operations. Several studies have also discussed the problem of coordinated PEV charging with different objectives such as the following: Optimization of PEV charging by reconfiguring the existing distribution network method [8]; Coordination of PEV charging to minimize power losses and improve voltage stability using the Real Time Smart Load Management algorithm [4]; Optimization of the PEV charging schedule using a heuristic method in the form of a genetic algorithm [1]. The aforementioned literatures discuss many aspects of PEV coordination problems in distribution systems. However, only a few studies have analyzed power losses and voltage stability as issues that are discussed at once. In addition, to the best of the knowledge of the authors of this thesis, no research has been conducted to analyze power losses as well as voltage deviation in the distribution network during PEV charging by considering optimization of PEV charging scheduling using metaheuristic methods.

This research focuses on the optimization of PEV charging coordination scheduling in distribution system by considering power losses and voltage deviation. This PEV charging coordination takes advantage of the PEV charging timings in the distribution system. To optimize the coordination of PEV charging, this study used a metaheuristic method called Binary Particle Swarm Optimization (BPSO) 
which were then compared with the Binary Gray Wolves Optimization (BGWO) method.

\section{PROBLEM FORMULATION}

\subsection{Objective Function}

The main focus of this research is to determine the optimal PEV charging schedule with the aim of minimizing power losses and voltage deviation. To simulate the coordination of PEV charging, the PEV charging time approach is carried out every 10 minutes (144 timeslots in 24 hours). The planned strategy for optimization of PEV charging coordination is optimization of PEV charging scheduling (in time intervals of every 10 minutes). The objective function of the optimization process to carry out the planned method is shown in Equation (1).

$$
\operatorname{minf}=\sum_{h}^{24}\left(\mathrm{P}_{\text {loss }}+V_{d}\right)
$$

$\mathrm{P}_{\text {loss }}$ is the ratio of power losses before and after PEV charging coordination. And $V_{d}$ is the maximum voltage deviation in the distribution system. The $\mathrm{P}_{\text {loss }}$ value is calculated from the comparison of the total power losses after and before the coordination is carried out as shown in Equation (2).

$$
\mathrm{P}_{\text {loss }}=\frac{P_{\text {loss }}^{\text {coord }}}{P_{\text {loss }}^{\text {uncord }}}
$$

The equation for power losses in the distribution system is shown in Equation (3).

$$
P_{\text {loss }}=\sum_{t=1}^{\text {timeslot }}\left(I_{j, t}^{2} \times R_{j}\right)
$$

The voltage deviation $\left(V_{d}\right)$ can be calculated from the difference between the nominal voltage value and the measured voltage. The smaller the value of the voltage deviation from the nominal voltage in a bus on the distribution network, the better the state of the voltage on the system. The calculation of the voltage deviation can be shown from Equation (4).

$$
V_{d}=\operatorname{Max}_{i=2}^{m}\left(\frac{V_{\text {rated }}-V_{i}}{V_{\text {rated }}}\right)
$$

$V_{\text {rated }}$ is the nominal voltage in the system which is 1.0 pu. $V_{i}$ is the voltage on the $i$-th bus, and $m$ is the total number of buses in the system.

\subsection{Constraints}

In the whole optimization process, some limitations or constraints on the system also need to be considered. Some of these constraints include:

\section{- Maximum Load}

$P_{\text {demand }}^{\max } \geq \sum_{i=2}^{n}\left(P_{\text {load }}+P_{P E V}\right)_{i}$

Description:

$\begin{array}{ll}n & : \text { the number of buses in the system } \\ P_{\text {load }} & : \text { housing load } \\ P_{P E V} & : \text { load of PEV } \\ i & : i \text {-th bus } \\ P_{\text {demand }}^{\text {max }} & : \text { maximum load on distribution } \\ & \text { transformer for } 24 \text { hours }\end{array}$

- Bus Voltage

$V_{\min } \leq V_{i} \leq V_{\max }$
Description:

$V_{\min }$ dan $V_{\text {max }}:$ the minimum and maximum voltage ranges allowed in the system

$V_{i} \quad:$ voltage on bus $i$

The value of the voltage limit range in this study is set at $\pm 10 \%$ of the nominal voltage, which is $0.9-1.1 \mathrm{pu}$.

\section{ALGORITHMS AND IMPLEMENTATION}

\subsection{Binary Particle Swarm Optimization (BPSO)}

In this study, Binary Particle Swarm Optimization (BPSO) was applied to complete the PEV charging coordination optimization method. The main difference between BPSO and PSO is only in the equation for changing the position of the particles. The equation for the velocity of each particle is shown in Equation (7) and the equation for the change in position for each particle is shown in Equation (8). All particles move in d-dimensional space.

$$
\begin{aligned}
\text { vel }_{i, d}^{t}= & w^{t} \text { vel }_{i, d}^{t}+c_{1} r_{1}\left(\text { pbest }_{i, d}^{t}-x_{i, d}^{t}\right)+ \\
& c_{2} r_{2}\left(\text { gbest }_{i, d}^{t}-x_{i, d}^{t}\right)
\end{aligned}
$$

Description:

vel $_{i, d}^{t} \quad$ : velocity of particle $i$ in dimension $d$ during time $t$

$x_{i, d}^{t} \quad:$ the position of particle $i$ in dimension $d$ during time $t$

$c_{1}$ dan $c_{2}$ : cognitive constant and social acceleration

$r_{1}$ dan $r_{2}$ : random values distributed between 0 dan 1

$$
\begin{aligned}
& x_{i, d}^{t+1}=\begin{array}{l}
0 \operatorname{rand}() \geq \operatorname{sig}\left(\operatorname{vel}_{i, d}\right) \\
1 \operatorname{rand}()<\operatorname{sig}\left(\operatorname{vel}_{i, d}\right)
\end{array} \\
& \operatorname{sig}\left(\operatorname{vel}_{i, d}\right)=\frac{1}{1+e^{-v e l_{i, d}}}
\end{aligned}
$$

In Equation (9), $\operatorname{sig}\left(\right.$ vel $\left._{i, d}\right)$ is the equation of the logistic function transformation and $\operatorname{rand}()$ is a random value distributed between " 0 " and " 1 ". BPSO was chosen in this study because of the nature of the solution required. BPSO provides a final solution in the form of binary "1" or " 0 ", which indicate charging and off-charging conditions in the PEV charging process, respectively.

The steps in the flow chart in Figure 1 can be used to determine the optimal coordination of PEV charging using the Binary Particle Swarm Optimization method. All constraints in the system are also considered at this stage. 


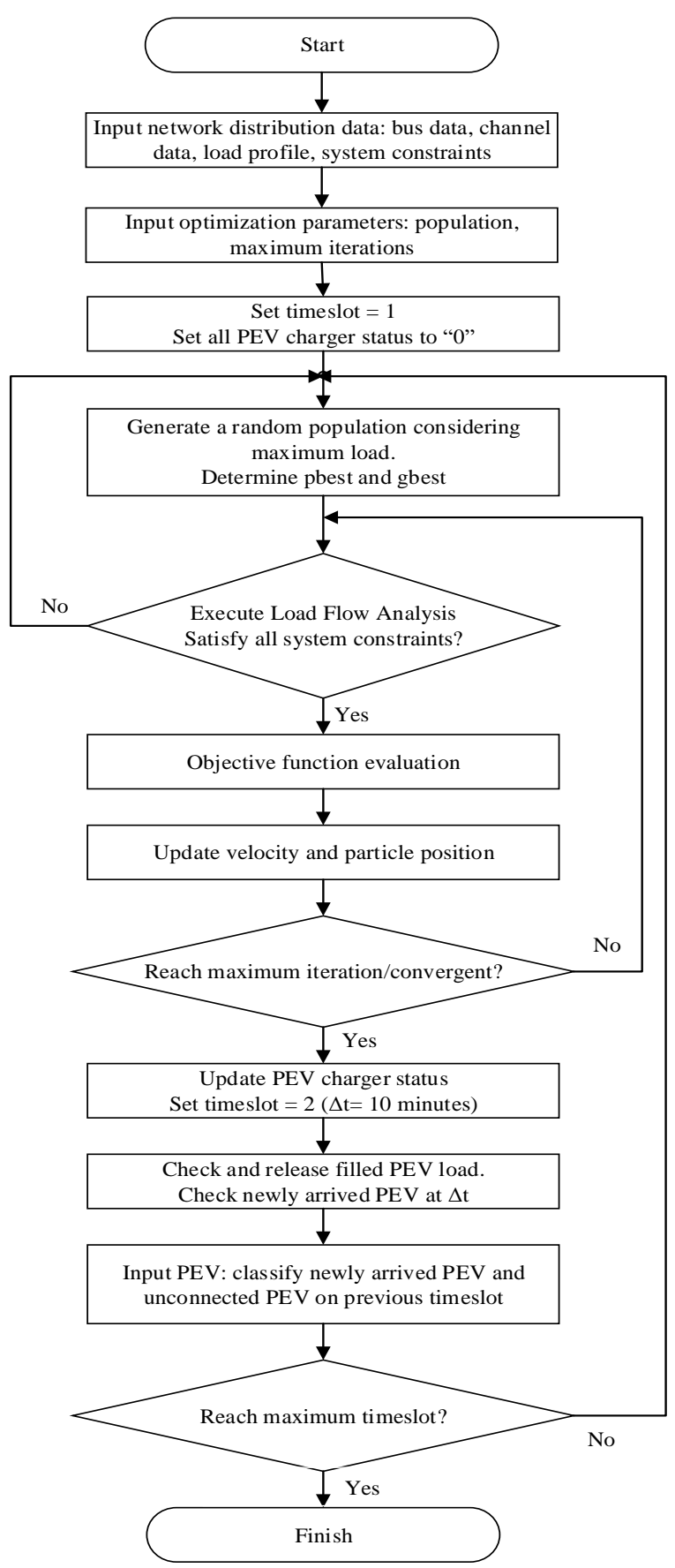

Figure 1. Flowchart of PEV charging coordination optimization using BPSO method

\subsection{Binary Grey Wolves Optimization (BGWO)}

The GWO algorithm is designed to solve continuous optimization problems. For binary optimization problems such as feature selection, a binary version of the GWO is required. In 2016, Emary developed the Binary Gray Wolves Optimization algorithm to solve the feature selection problem. The equation for the change in position in the BGWO is shown in Equation (10).

$$
X(t+1)=\text { Crossover }\left(Y_{1}, Y_{2}, Y_{3}\right)
$$

Crossover $\left(Y_{1}, Y_{2}, Y_{3}\right)$ is a crossover operation between solutions, while $Y_{1}, Y_{2}$, and $Y_{3}$ are binary vectors affected by the movement of the alpha, beta, and delta wolves. In this BGWO, the values of $Y_{1}, Y_{2}$, and $Y_{3}$ are calculated using Equation (11) to Equation (13).

$$
\begin{gathered}
Y_{1}^{d}=\left\{\begin{array}{c}
1, \text { if }\left(X_{\alpha}^{d}+\text { bstep }_{\alpha}^{d}\right) \geq 1 \\
0, \text { otherwise }
\end{array}\right. \\
Y_{2}^{d}=\left\{\begin{array}{c}
1, \text { if }\left(X_{\beta}^{d}+\text { bstep }_{\beta}^{d}\right) \geq 1 \\
0, \text { otherwise }
\end{array}\right. \\
Y_{3}^{d}=\left\{\begin{array}{c}
1, \text { if }\left(X_{\delta}^{d}+\right.\text { bstep } \\
0, \text { otherwise }
\end{array}\right) \geq 1
\end{gathered}
$$

In Figure 2, a PEV charging optimization flow chart is shown using the Binary Gray Wolves Optimization method. This step can be used to determine the optimal coordination of PEV charging with the aim of minimizing power losses and voltage deviations simultaneously. All constraints in the system are also considered at this stage.

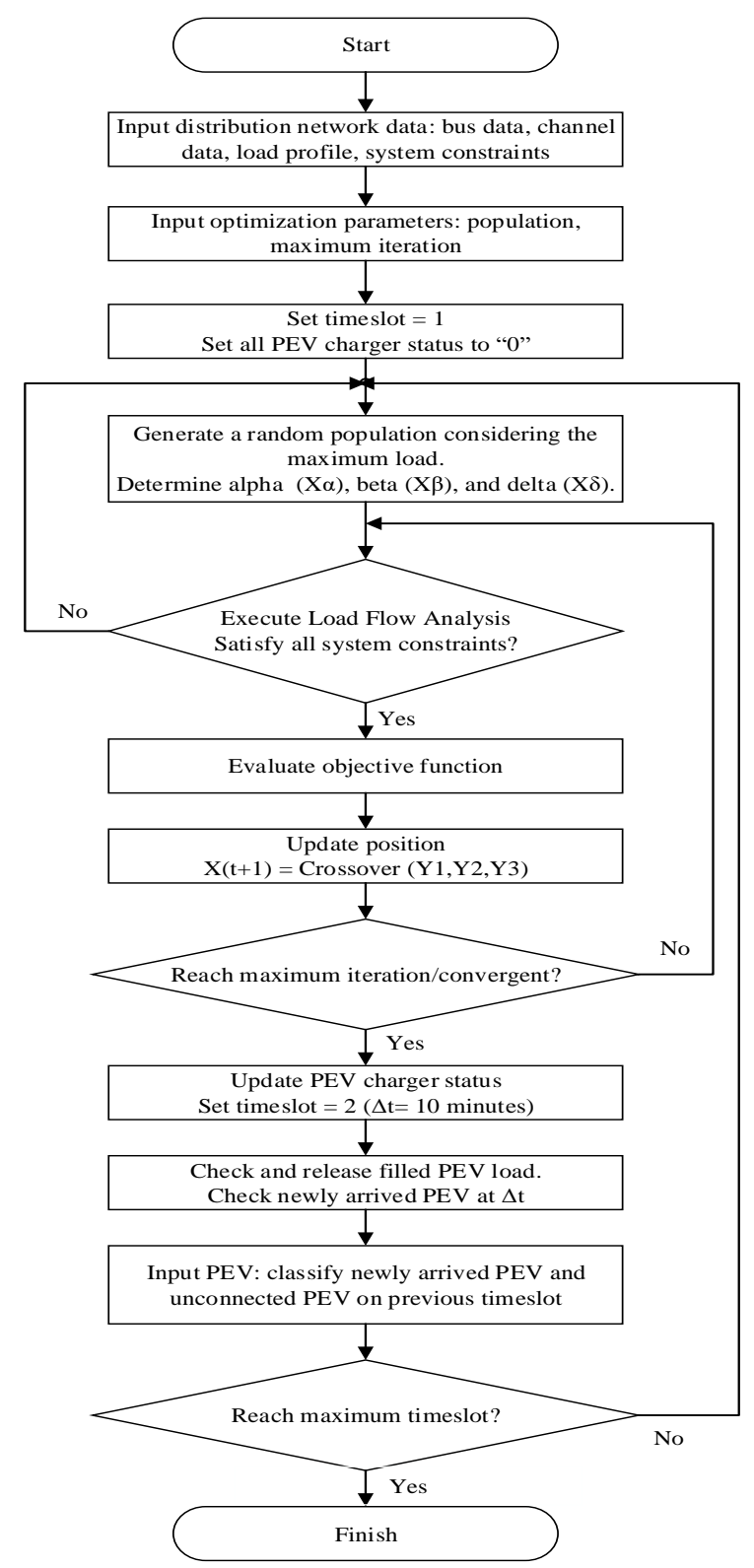

Figure 2. Flowchart of PEV charging coordination optimization using BGWO method 


\section{RESULTS AND DISCUSSION}

\subsection{Existing System Flow Analysis Prior to Optimization of PEV Charging}

\section{Coordination}

The power flow analysis of the existing system is carried out when there is already PEV penetration but the optimization process for PEV charging coordination has not been carried out. In this study, 4 cases of PEV penetration will be analyzed. Namely PEV penetration in the system for penetration values of $20 \%, 40 \%, 60 \%$, and $80 \%$. This PEV penetration value is based on the added value of the total load in the Pujon feeder which is burdened by the existing household load. For the loading value of 1 unit of PEV in accordance with the limitations of this research problem, namely PEV with the maximum required charging power specification of $4.4 \mathrm{kVA}$. PEV are distributed according to buses located in densely populated areas or buses with sufficient transformer capacity to accommodate the additional PEV load. The results of the analysis of the existing load power flow after the penetration of PEV in the 24-hour time interval is shown in the graphs shown in Figures 3 to 5.

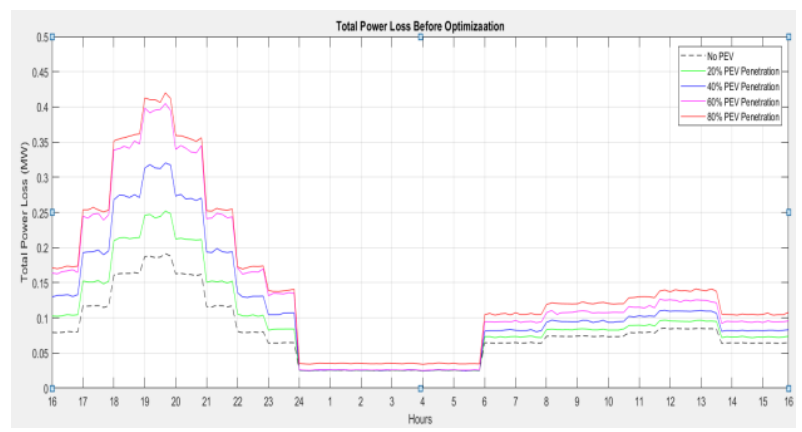

Figure 3. Total power loss before optimization

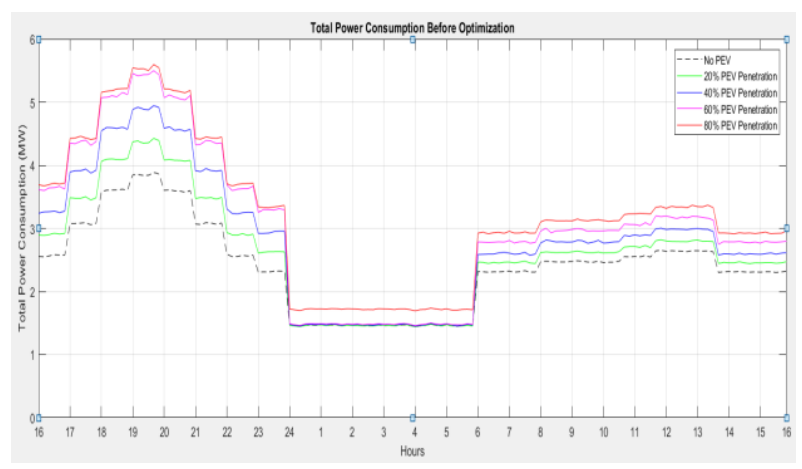

Figure 4. Total power consumption before optimization

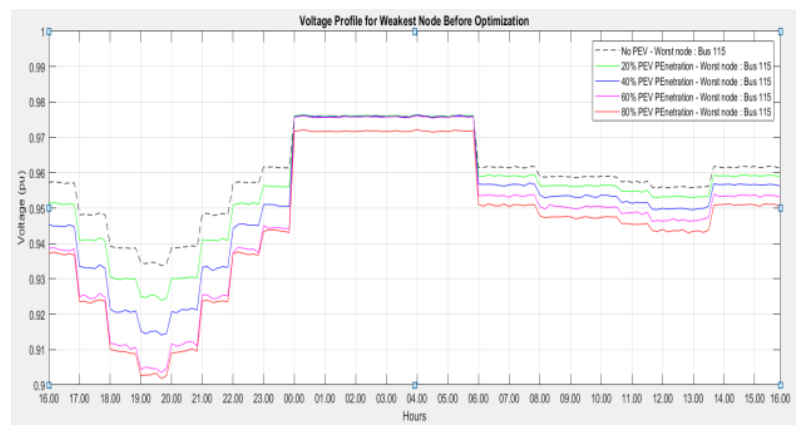

Figure 5. Voltage profile for weakest node before optimiztion
In this scenario, the PEV charging process starts as soon as the PEV is connected to a charging device. PEVs that are connected within a certain time are filled in randomly regardless of the constraints in the existing distribution system. Therefore, the existing system faces more problems such as overload in the distribution system, high power losses and voltage degradation. The total value of power losses in a 24-hour time interval is shown in Figure 3. It was found that this random and uncoordinated PEV charging process causes power losses to increase, especially during peak hours, the maximum load occurs from 20.00 to 23.00 . With the highest value of power losses at $80 \%$ penetration of $0.42 \mathrm{MW}$ occurring at 19.40. Figure 4 shows that the total loading power also increases when the PEV penetrates. The total loading power is relatively high in the presence of PEV penetration. The highest total loading power value occurs when the penetration of $80 \% \mathrm{PEV}$ is $5.6 \mathrm{MW}$ at 19.40 . In Figure 5 it is shown when the PEV penetration is at $60 \%$ and $80 \%$, with the lowest stress value of $0.9 \mathrm{pu}$. Meanwhile, at $20 \%$ and $40 \%$ PEV penetration, the lowest stress values were still within the permissible limits, which were 0.92 and 0.91 pu.

The value of the power loss after the penetration of the PEV load can be used as a reference for the optimization of PEV charging scheduling coordination considerations. This is because when there is penetration of the PEV load, the existing system conditions cause an increase in the value of power losses and an indication of a lower voltage profile, with an average of below 0.95 p.u. Meanwhile, the permissible voltage profile according to SPLN 1: 1995 is $0.90 \mathrm{p} . \mathrm{u} \leq$ Vbus $\leq 1.1$ p.u.

\subsection{Optimization of PEV Charging Coordination with Binary Particle Swarm Optimization Algorithm}

The BPSO algorithm is the first algorithm used in the optimization of PEV charging coordination. The first step after entering the load data and channel data into Newton Raphson's calculations was to determine the penetration limit of the PEV distributed into the system. The second step was to enter the initial parameters used in the optimization process using BPSO. The third step was to run optimization in 4 cases of PEV penetration to obtain an analysis of conditions in the system in sharing cases of PEV penetration.

To overcome the adverse effects of uncoordinated PEV charging on the distribution network, a real-time PEV charging coordination approach based on 10-minute intervals was proposed in this study. When the PEV is connected to a charger, the PEV will not be charged immediately as long as the charging decision is not taken by the control center. The optimally implemented BPSO algorithm schedules the arriving PEV and can meet all system constraints. This coordination strategy is more advantageous for the existing distribution system compared to the case of uncoordinated PEV charging. Figures 6 to 8 show the results of the system power flow analysis after optimization using BPSO. 


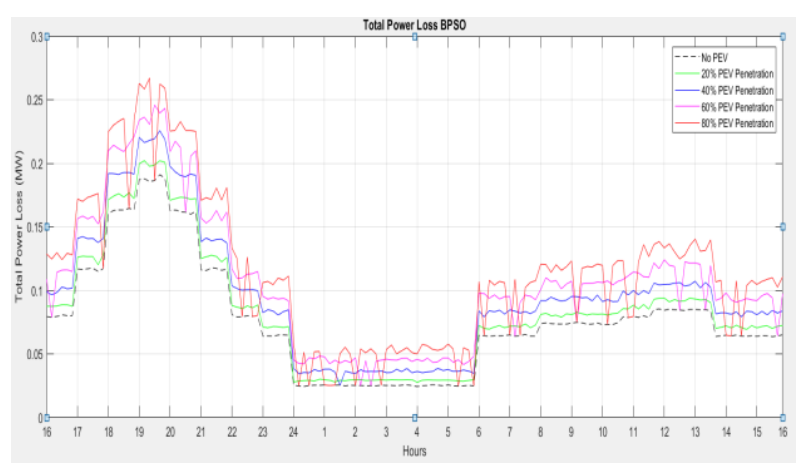

Figure 6. Total power loss system using BPSO algorithm

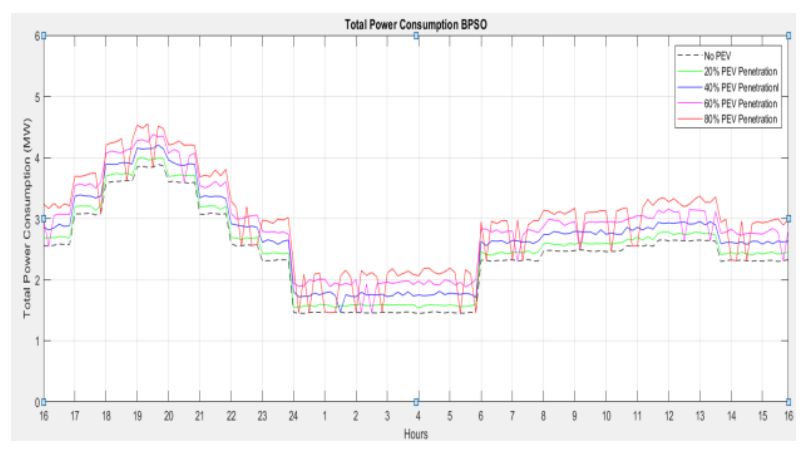

Figure 7. Total power consumption using BPSO algorithm

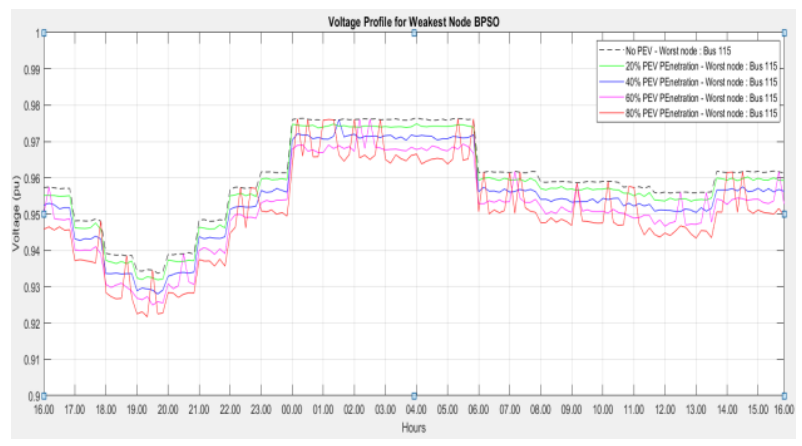

Figure 8. Voltage profile for the weakest node using BPSO algorithm

Figure 6 shows that the highest value of power losses occurs when the $80 \%$ PEV penetration can be reduced from $0.42 \mathrm{MW}$ to $0.26 \mathrm{MW}$. Likewise, the total power consumption and voltage on the system can also decrease after optimization using BPSO. This is shown in Figure 7, with the highest total power consumption of $4.51 \mathrm{MW}$, while and Figure 8 shows the lowest voltage of $0.92 \mathrm{pu}$.

\subsection{Optimization of PEV Charging Coordination with Binary Gray Wolves Optimization Algorithm}

The BGWO algorithm is the second algorithm used in the optimization of PEV charging coordination. The first step after entering the load data and channel data into Newton Raphson's calculations was to determine the penetration limit of the PEV distributed into the system. The second step was to enter the initial parameters used in the optimization process using BGWO. The third step was to run optimization in 4 cases of PEV penetration to obtain an analysis of conditions in the system in sharing cases of PEV penetration.

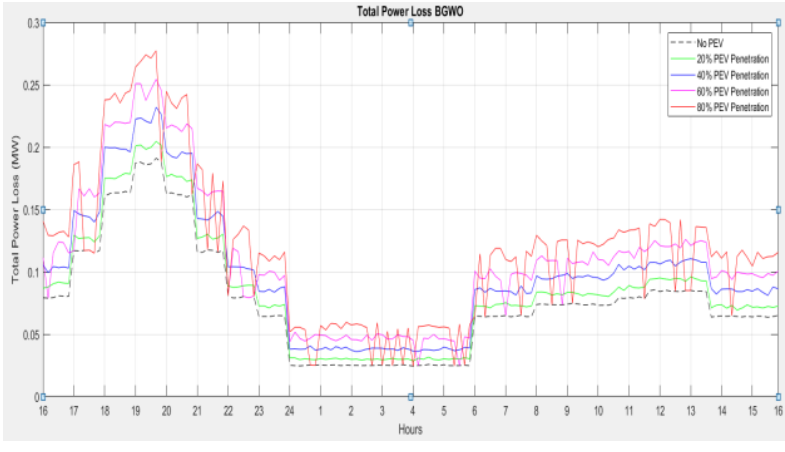

Figure 9. Total power loss system using BGWO algorithm

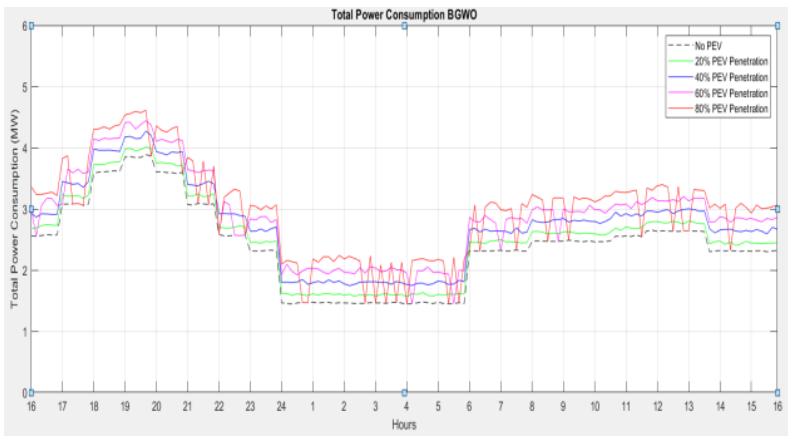

Figure 10. Total power consumption using BGWO algorithm

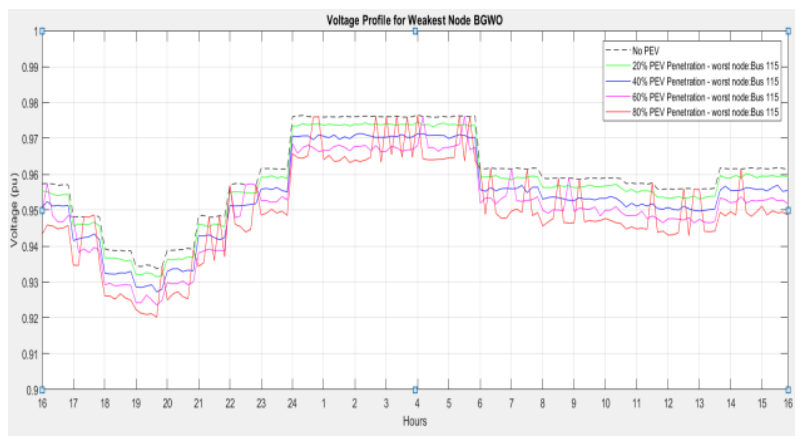

Figure 11. Voltage profile for the weakest node using BGWO algorithm

Figure 9 shows that the value of power losses that occur during PEV penetration can be reduced unlike before the optimization process. In the case of $80 \%$ penetration, the power losses can be reduced to $0.28 \mathrm{MW}$. The total power consumption in Figure 10 shows that it has also decreased to 4.61 MW. Likewise, the voltage value in all cases of PEV penetration also decreased, as in $80 \%$ penetration the voltage value at the worst node became $0.92 \mathrm{pu}$.

\subsection{Comparative Analysis of BPSO and BGWO Algorithms}

The simulation results of the PEV charging coordination power flow analysis using the BPSO and BGWO algorithms are summarized in Table 1. It can be seen that the total power losses can be reduced for all PEV penetration levels when the PEV charging coordination optimization process is carried out. It can also be observed that the overall voltage profile of the network increases. In addition, in terms of power consumption and power losses, network performance improved. 
Table 1. Impact of PEV charging on distribution systems in various cases

\begin{tabular}{|l|c|c|c|}
\hline \multicolumn{1}{|c|}{ Scenario } & PEV Penetration(\%) & $\Delta \mathrm{V}(\%)$ & Increase in $\operatorname{loss}^{2}(\%)$ \\
\hline No PEV & 0 & 6,63 & 0 \\
\hline \multirow{3}{*}{ Uncoordinated Charging } & & & \\
& $20 \%$ PEV penetration & 7,61 & 24,16 \\
& $40 \%$ PEV penetration & 8,58 & 40,36 \\
& $60 \%$ PEV penetration & 9,65 & 52,80 \\
& $80 \%$ PEV penetration & 9,82 & 54,51 \\
Coordinaed Charging using BPSO & $20 \%$ PEV penetration & 6,81 & 5,45 \\
& $40 \%$ PEV penetration & 7,20 & 15,35 \\
& $60 \%$ PEV penetration & 7,41 & 20,24 \\
& $80 \%$ PEV penetration & 7,75 & 27,20 \\
Coordinaed Charging using BGWO & & & \\
& $20 \%$ PEV penetration & 6,86 & 6,58 \\
& $40 \%$ PEV penetration & 7,28 & 17,62 \\
& $60 \%$ PEV penetration & 7,65 & 24,89 \\
& $80 \%$ PEV penetration & 7,98 & 31,09 \\
\hline
\end{tabular}

Increase in loss compare to no pev case

\section{CONCLUSION}

In distribution systems, uncoordinated and random PEV charging activity results in overloading of distribution transformers, severe voltage deviation, and significantly increased system power losses. Therefore, the main objective of this study is to develop an optimal PEV charging process. The results of this study show that the use of the BPSO and BGWO algorithm methods is able to produce PEV charging coordination with significantly decreased power losses and voltage deviations compared to uncoordinated PEV filling.

\section{REFERENCES}

[1] Alonso, M.; Amaris, H.; Germain, J.; and Galan, J. 2014. Optimal Charging Scheduling of Electric Vehicles in Smart Grids by Heuristic Algorithms. Energies, 7, 2449.

[2] Browne D, O'Mahony M, Caulfield B. 2012. How should barriers to alternative fuels and vehicles be classified and potential policies to promote innovative technologies be evaluated? Journal of Cleaner Production, 35:140-151.

[3] Clement-Nyns, Kristien . 2010. Impact of Plug-in Hybrid Electric Vehicles on the Electricity System. Belgium : Katholieke Universiteit Leuven, Departement Elektrotechniek

[4] Deilami S, Masoum AS, Moses PS, Masoum MAS. 2011. Realtime coordination of plug-in electric vehicle charging in smart grids to minimize power losses and improve voltage profile. IEEE Transactions on Smart Grid; 2(3):456-467.
[5] E. Emary, Hossam M. Zawbaa, Aboul Ella Hassanien. 2015. Binary Gray Wolf Optimization Approaches for Feature Selection. Elsevier Neurocomputing International Journal.

[6] Garcia-Valle, R. and Lopes, J. P. 2013. Electric Vehicle Integration into Modern Networks. Springer. pp. 24-28. 63-64.

[7] J. Kennedy and R. Eberhart. 1995. Particle Swarm Optimization. IEEE Int. Conf on Neural Networks, Perth, Australia, 1942-1948.

[8] K. Naidu , M. A. Muhammad, H. Mokhlis, M. Sufyan, and A. Amin. 2019. Optimal coordination of EV charging with network reconfiguration. Jawa Barat, Indonesia : AIP Conference Proceedings 2129.

[9] Knezovic, Katarina. 2016. Phase-wise enhanced voltage support from electric vehicles in a Danish low-voltage distribution grid. Denmark : Center For Electric Power and Energy, Department of Electrical Engineering, Technical University of Denmark.

[10] Kongjeen,Yuttana and Bhumkittipich, Krischonme. 2018. Impact of Plug-in Electric Vehicles Integrated into Power Distribution System Based on Voltage-Dependent Power Flow Analysis. Thailand : Department of Electrical Engineering, Rajamangala University of Technology.

[11] Markel, T. and Simpson, A. 2006. Plug-in hybrid electric vehicle energy storage system design. In: 6th Advanced Automotive Battery Conference. Baltimore, MD.

[12] Mirjalili, S., Mirjalili, S.M.,and Lewis, A. 2014. Grey Wolf Optimizer. Advance Engineering Software: 69. Elsevier.

[13] Saelee, S. 2016. Optimal Placement of Ev Charging Station Considering The Road Traffic Volume And Ev Running Distance. Thailand : Sirindhorn International Institute Of Technology.

[14] Stevenson, William D., Granger, John J., 1994. Power System Analysis, McGraw-Hill International Edition., New York.

[15] R. D. Zimmermann , C.E. Murillo-Sánchez , R. J. Thomas. 2011. MATPOWER: Steady- State Operations, Planning and Analysis Tools for Power Systems Research and Education. Power Systems, IEEE Transactions on, vol. 26, no. 1, pp. 12-19.

[16] Tann, K. M., Ramachandaramurthy, V. K. and Yong, J. Y. 2015. Integration of electric vehicles in smart grid: A review on vehicle to grid technologies and optimization techniques. Malaysia : Department of Electrical Engineering, Universiti Tenaga Nasional Malaysia.

[17] Yuttana, K.; Wannawit, J.; Krischonme, B.; Nadarajah, M. 2018. Estimation of the Quick Charging Station for Electric Vehicles based on Location and Population Density. International Journal of Intelligent Engineering \& Systems. 